\title{
Immune Response of Mormon Crickets That Survived Infection by Beauveria bassiana
}

\author{
Robert B. Srygley and Stefan T. Jaronski \\ Northern Plains Agricultural Research Laboratory, USDA-Agricultural Research Service, 1500 N. Central Avenue, \\ Sidney, MT 59270, USA \\ Correspondence should be addressed to Robert B. Srygley, robert.srygley@ars.usda.gov
}

Received 1 June 2010; Accepted 29 July 2010

Academic Editor: Michel Lecoq

Copyright ( 2011 R. B. Srygley and S. T. Jaronski. This is an open access article distributed under the Creative Commons Attribution License, which permits unrestricted use, distribution, and reproduction in any medium, provided the original work is properly cited.

\begin{abstract}
Beauveria bassiana (Fungi: Ascomycota) is an entomopathogenic fungus that serves as a biological control agent of Mormon crickets Anabrus simplex Haldeman (Orthoptera: Tettigoniidae) and other grasshopper pests. To measure the dose-dependent response of Mormon crickets to fungal attack, we applied B. bassiana strain GHA topically to adults using doses of $5.13 \times 10^{4}$ to $1.75 \times 10^{6}$ conidia in sunflower oil, with oil only as a control. After three weeks, we assessed the survivors' hemolymph for fungal cells, active phenoloxidase (PO), and lysozyme. Mortality increased and body mass of survivors decreased with conidial dose. survivors' PO activity was elevated to the same level independent of dose. Those with fungal cells visible in their hemolymph did not differ in PO activity from those with clear hemolymph. We conclude that circulating PO may be an important enzymatic defense against Beauveria infection and that it is associated with attempted clearing of Beauveria blastospores and hyphae from Mormon cricket hemolymph.
\end{abstract}

\section{Introduction}

Nomadic insects risk contact with fungal pathogens [1]. Mormon crickets, a long-horned grasshopper or katydid, form bands and march across western United States grasslands seeking food, salt, and oviposition sites (Figure 1, $[2,3])$. Wingless, they must walk, which increases the risk of contacting insect-pathogenic ascomycetous fungi, such as Beauveria spp. and Metarhizium spp., on plants or soil [4]. These fungal pathogens occur naturally, but some strains, such as the commercial Beauveria bassiana GHA, may be applied artificially as control agents.

The ability of the fungus to infect an insect depends on its ability to adhere and penetrate the exoskeleton, resist the insect's hemolymph-borne defenses, and grow rapidly [5]. The conidium adheres to the cuticle and germinates to penetrate the exoskeleton with a combination of mechanical pressure and a cocktail of lytic enzymes. The insect may respond to the wounding with local induction of the phenoloxidase (PO) cascade, resulting in production of toxic quinones and cuticular melanization. Following penetration into the hemolymph, the fungus grows as a yeast-like blastospore or as short lengths of vegetative hyphae. Insect defenses include encapsulation of the fungus by granulocytes and plasmatocytes (both circulating hemocytes) and formation of a nodule that may be melanized [6]. Grasshoppers may also respond with behavioral fevers, elevating body temperature to inhibit fungal growth $[7,8]$. Mormon crickets do not demonstrate behavioral fever per se; their preferred body temperatures are $34-37^{\circ} \mathrm{C}$ [9], above the upper thermal limit for most entomopathogenic Ascomycetes. Death of the host may result from competition with the pathogen for nutrients, mechanical damage resulting from hyphal growth, and fungal toxins [5].

The humoral defenses of insects to pathogenic fungi have only been investigated in a handful of species. Metarhizium infection may result in declining hemolymph protein and $\mathrm{PO}$ titres over the course of the infection until death (Schistocerca gregaria [10], Locusta migratoria [6]) whereas Beauveria infection increases active PO levels (Melanoplus sanguinipes [11], Spodoptera exigua [12]). Lysozyme activity may decline (Schistocerca gregaria [10]) or remain unchanged 
(Spodoptera exigua [13]). In this paper, we investigate circulating PO and lysozyme titres in adult Mormon crickets that have successfully defended themselves against invasion from topically applied Beauveria bassiana strain GHA. On rangeland and crops, control agents are frequently not applied until Mormon crickets have reached the adult stage because the public demand for control is greatest when Mormon crickets have banded together and migrated from natal sites into habitats where they interfere with human activities.

\section{Materials and Methods}

2.1. Fungal Conidia. The B. bassiana conidia were obtained from Laverlam International (Butte, Montana, USA.) as a dry technical grade conidial powder. Conidial viability was determined by plating aqueous conidial suspensions onto quarter-strength potato dextrose agar, incubating the fungi at $28^{\circ} \mathrm{C}$ for $18-20 \mathrm{hr}$, then examining with $400 \mathrm{x}$ phase-contrast microscopy for germination. A conidium was considered germinated and thus viable if a germination peg was visible. A concentrated stock suspension in sunflower oil was prepared from the dry conidia, and the concentration was determined by hemocytometer counts of kerosenediluted samples and adjusted for conidial viability. Working dilutions were prepared from the two concentrates using positive displacement pipettes, and the exact concentrations were determined by hemocytometer counts of kerosenediluted samples. All conidia concentrations are viable conidia per unit volume.

2.2. B. Bassiana Dose Response. Adult Mormon crickets were collected at Lodge Grass, Montana on July 17, 2007, and fungal treatments were topically applied on July 24 (1st replicate) and July 25 (2nd replicate) to the base of the first leg, including the following fungal doses suspended in $1 \mu \mathrm{l}$ sunflower oil: $1.75 * 10^{6}, 1.07 * 10^{6}, 3.54 * 10^{5}, 1.13 * 10^{5}$, or $5.13 * 10^{4}$ conidia/ $\mu \mathrm{l}$ B. bassiana strain GHA or a control treatment of only sunflower oil. Survivorship was measured over 21 days at $28^{\circ} \mathrm{C}$.

2.3. Immunity Assays and Total Protein. After three weeks, we drew hemolymph from the surviving adults (five males and five females for each treatment, fewer if there were not enough survivors) to assess spontaneously active PO, lysozyme-like activity, and total hemolymph protein. We measured the body mass of each cricket to the nearest mg with an Ohaus microbalance (model AV53) and then punctured the arthrodial membrane at the base of the hind leg of each insect with a 26 gauge hypodermic needle so that it exuded hemolymph. A total of $14 \mu \mathrm{L}$ of hemolymph was collected into a capillary tube, with a second puncture performed when necessary. For assays of PO activity and total hemolymph protein, the hemolymph was diluted $1: 50$ with phosphate buffered saline (PBS) solution and frozen at $-20^{\circ} \mathrm{C}$. An additional $10 \mu \mathrm{L}$ hemolymph diluted $1: 10$ with PBS was stored at $-20^{\circ} \mathrm{C}$ for subsequent measuring of lysozyme activity. For ten insects, we did not collect sufficient blood for all of the tests.

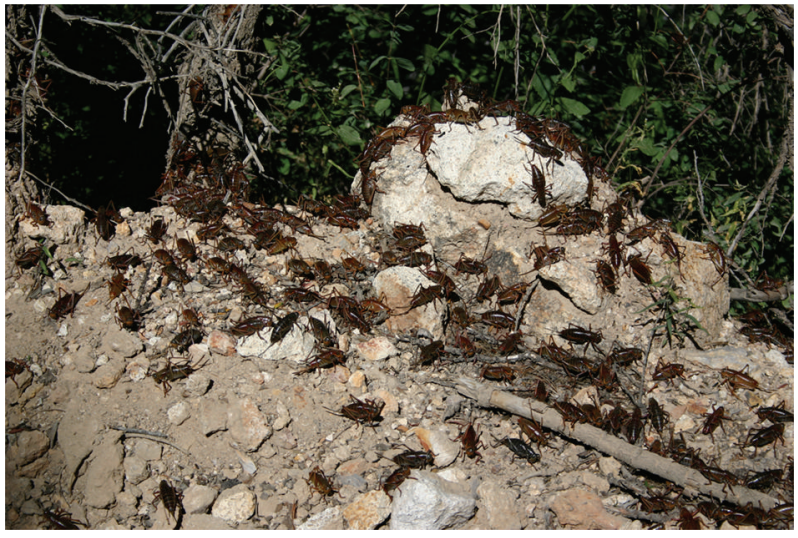

FIgure 1: Migrating Mormon crickets basking near Jarbidge, Nevada in July 2009.

To measure PO activity, we followed the protocol of Wilson et al. [8]. Samples of thawed hemolymph diluted in PBS were centrifuged $\left(4^{\circ} \mathrm{C}, 10,300 \mathrm{rpm}\right.$ for 10 minutes) and activated with $10 \mathrm{mM}$ dopamine solution. The plate was loaded into a temperature-controlled BioTek microplate reader $\left(25^{\circ} \mathrm{C}\right)$, and absorbance at $492 \mathrm{~nm}$ was read between 5 and 15 minutes. If sample absorbance was linearly related with time, we calculated mean $\mathrm{V}$ (change in absorbance $\mathrm{min}^{-1}$ ). One unit PO activity per $\mathrm{ml}$ hemolymph is defined as the amount of enzyme resulting in a 0.001 increase in absorbance.

To measure lysozyme-like antibacterial activity, a turbidimetric method was used, following the protocol of de Azambuja et al. [14]. Thawed and PBS-diluted hemolymph was added to a well with suspended gram-positive bacteria cells Micrococcus lysodeikticus (Worthington). Clearing of the well was compared to a serial dilution of egg-white lysozyme (Sigma) added to the bacteria suspension. The plate was loaded into a temperature-controlled Biotek microplate reader $\left(25^{\circ} \mathrm{C}\right)$, and absorbance at $450 \mathrm{~nm}$ was read between 10 and 30 minutes. If the sample absorbance was linearly related with time, we would calculate mean $\mathrm{V}$. When sample activity fell below $6.5 \mu \mathrm{g} \mathrm{ml}^{-1}$, the sample was excluded because the standards showed that the data were unreliable when samples were this weak.

We measured total hemolymph protein in $\mathrm{mg}$ protein $\mathrm{ml}^{-1}$ hemolymph with a Total Protein Kit, Micro (Sigma) compared to a serial dilution of the human albumin standard.

2.4. Verifying Infection. An additional $10 \mu \mathrm{L}$ of hemolymph collected as described above was smeared on a slide and stained with a drop of lactofuchsin. Hemolymph samples were scanned at $400 \mathrm{x}$, using dark-field, phase-contrast microscopy, for hyphae and blastospores.

2.5. Statistical Analyses. To analyze the B. bassiana dose response data, we combined the data from both replicates because Fisher's Exact Tests indicated no significant differences between the replicates at each dose. The combined data 
TAble 1: Pathogenicity of Beauveria bassiana strain GHA for adult Anabrus simplex based on mortalities 21 days after topical application.

\begin{tabular}{lcccc}
\hline $\begin{array}{l}\text { LD50 } \\
\text { (conidia/insect) }\end{array}$ & $\begin{array}{c}95 \% \text { Confidence } \\
\text { Limits } \\
\text { (conidia/insect) }\end{array}$ & Slope (S.E) & $\begin{array}{c}\text { Chi- } \\
\text { Square } \\
(P)^{*}\end{array}$ & $g^{* *}$ \\
\hline $6.46 \times 10^{5}$ & $\begin{array}{c}3.97 \times 10^{5}- \\
1.275 \times 10^{6}\end{array}$ & $\begin{array}{c}0.885 \\
(0.171)\end{array}$ & $6.745(.08)$ & 0.144 \\
\hline
\end{tabular}

${ }^{*}$ Chi-square of heterogeneity: measures goodness of fit to the weighted regression line with $P>.05$ indicating a good fit of the data to the line. D.F. $=5$

$* * g$ is the index of regression significance.

were then subjected to probit analysis using LDP Line (LdP Line, 2000 by Ehab Mostofa Bakr, Cairo, Egypt). Lysozyme and $\log _{10}$-transformed PO were normally distributed. Applying ANCOVA, we covaried the dependent variables with body mass and tested them for effects of replicate, sex and fungal dose (sample sizes in order of dosage from highest to lowest: $n=2,8,9,10,10$, and 10 for the 1st replicate and $n=3,5,8,6,9$, and 10 for the 2nd). Body mass was not a significant covariate, and so here we report the results from the three-way ANOVA's. Only for the males did the total protein meet the assumptions for parametric statistical analyses, and so we applied nonparametric statistics to data for the females.

Data for PO and total protein were normally distributed after $\log _{10}$ transformations. Lysozyme activity was normally distributed after squaring the data. Applying ANCOVA, we covaried the dependent variables with body mass and tested them for effects of sex and fungal treatment. However, body mass was not a significant covariate, and so we simplified the analysis and reported the two-way ANOVA's.

\section{Results}

Mortality at 21 days ranged from $22 \%$ to $80 \%$ and increased with the dose of $B$. bassiana applied to the cuticle (Table 1) with an LD50 estimate of $6.46 \times 10^{5}$ conidia per insect.

For survivors, mean body masses of replicates were significantly different $(P=.038)$, and those for all treatments except one were significantly less than that for controls, but there was no difference in body mass among B. bassiana doses (Figure 2(a)). Log PO differed significantly between replicates and dose $(P=.0015$ and $P=.0048$, resp. $)$ whereas it did not differ between the sexes $(P=.80)$. In a post hoc comparison among the means, Mormon crickets treated with B. bassiana had greater PO activity than uninfected controls, but none of the fungal treatments differed from one another (Figure 2(b)). The second replicate also had significantly greater lysozyme activity than the first $(P=.030)$ whereas sex and dose did not have significant effects $(P=.81$ and $P=.57$, resp.). Within males, total protein was proportional to body mass $(P<.0001)$, and insects in the second replicate had significantly greater total protein than those in the first $(P=.0025$, resp. $)$, but fungal treatment was not a significant factor affecting total protein $(P=.635)$. Females in the second replicate also had significantly greater total protein

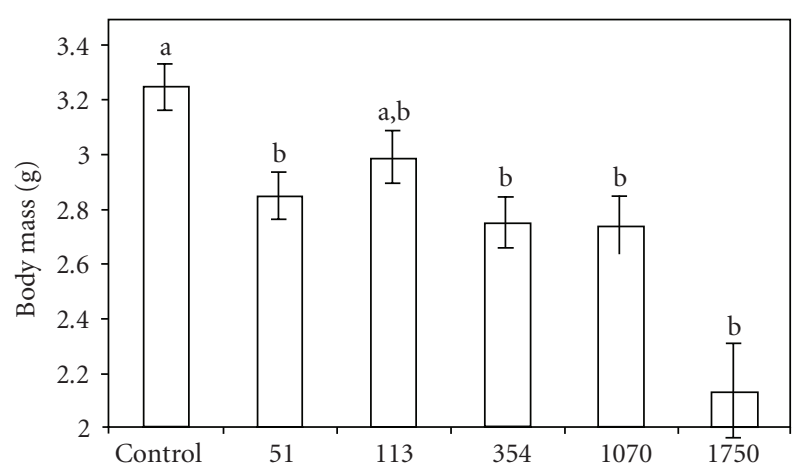

(a)

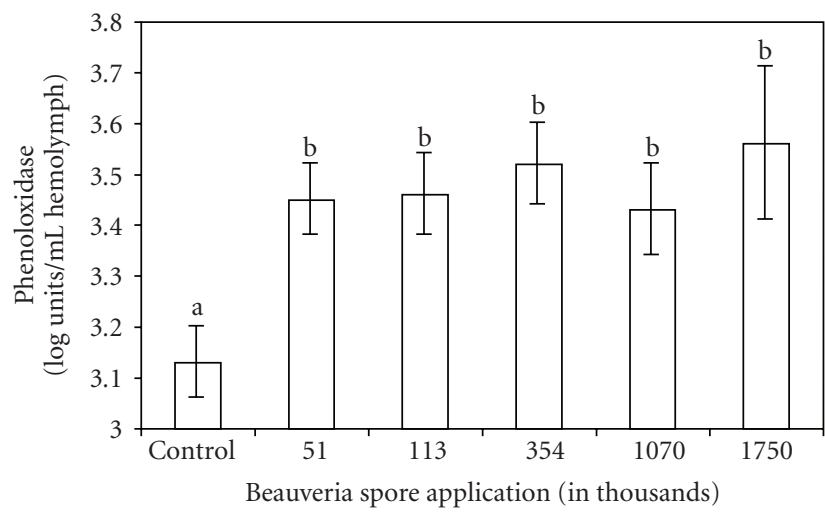

(b)

Figure 2: (a) Body mass and (b) phenoloxidase (PO) activity of adult Mormon crickets relative to the dose of Beauveria bassiana applied. Means and standard errors of the two replicates are shown with significantly different means in post hoc comparisons indicated by different letters.

than those in the first replicate (Wilcoxon test, $S=423, z=$ 2.02, $P=.043$ ), but fungal treatment was not a significant factor affecting total protein within replicates $(P>.60)$.

\section{Discussion}

Mormon crickets responded to $B$. bassiana infection with an increase in PO. Beauveria infection also increased active PO levels in the grasshopper Melanoplus sanguinipes and the army cutworm Spodoptera exigua [12]. Gillespie and Khachatourians [11] found that after topical application of $10^{8}$ conidia to M. sanguinipes, PO levels increased 3.8 times in males peaking at 3 days postinfection and 8.3 times in females peaking on the first day postinfection. In $M$. sanguinipes after 5 days, $\mathrm{PO}$ levels had returned to near control levels in males, but in females remained more than twice that of controls. Our applied doses were lower, and more of the Mormon crickets survived the application. At 21 days, PO levels remained higher in Beauveria-treated Mormon crickets relative to controls. We did not observe a difference in PO levels between the sexes for either controls or those that survived fungal application. Surprisingly, PO titres of Beauveria-treated survivors were independent of the dose applied. 


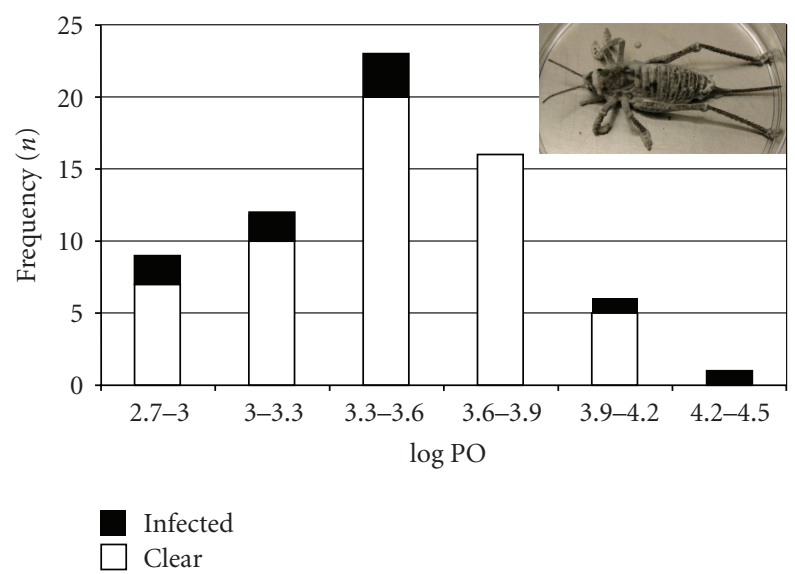

FIgURe 3: Phenoloxidase (PO) activity of survivors with fungal cells visible in their hemolymph (infected) and that of survivors with clear hemolymph. Inset: dark brown adult female Mormon cricket with white Beauveria sporulating on its cuticle.

Total circulating protein concentrations did not differ between treatments in males or females. In Melanoplus sanguinipes, protein concentrations of males and females peaked $30 \%$ above that of controls within three days of infection, but returned to the same level as controls by day five post infection [11].

The second replicate had higher PO, lysozyme, and total protein titers than the first. Adults were collected from the same location on the same day and treated only a day apart to make replicates as similar as possible, and thus the reason for these differences is not known. Body mass of individuals did not differ significantly between control groups $(n=20, P=$ .38 ), and so individuals in the second replicate were probably in no better overall condition to defend against the fungus than the first. Indeed, the average mass of the first replicate was $6 \%$ greater than that of the second replicate-the opposite of what one would expect if condition were a factor.

Beauveria-treated individuals lost on average $17 \%$ of their mass relative to controls. Reduced food consumption is the most likely cause. Schistocerca gregaria eats less when infected with Metarhizium [15], and Manduca sexta stops feeding altogether [16]. However, an increase in metabolism with infection could also increase mass loss. Metabolic rate might increase because the Mormon cricket is fending off the infection or as a result of the contribution of the growing fungus. Reduced nutrient absorption from the gut or greater water loss might also contribute to mass loss and warrant further study.

PO activity of survivors with fungal cells visible in their hemolymph did not differ significantly from those with clear hemolymph ( $n=57$ fungus absent, $n=9$ fungus present, Welch ANOVA $F=0.06$, d.f. $=1,9, P=.81$, Figure 3 ). We conclude that circulating PO may be an important enzymatic defense against Beauveria infection and that it is associated with attempted clearing of Beauveria blastospores and hyphae from the hemolymph of Mormon crickets.

Beauveria bassiana infection did not affect lysozyme activity in the Mormon crickets. Hence, elevation of PO did not result in an elevation of antibacterial activity in an allor-none manner. Lysozyme activity declined with Beauveria infection in the desert locust Schistocerca gregaria [10] but remained unchanged in the army cutworm Spodoptera exigua [13].

In some Mormon cricket bands, migrating individuals seek protein [3], and protein ingestion is associated with an increase in PO activity [17]. Thus, protein deficiency evident in migratory bands is also likely to result in greater susceptibility to and more efficacious application of $B$. bassiana GHA.

\section{Acknowledgments}

The authors thank Rob Schlothauser, USDA-Agricultural Research Services, for help with fungal infections and Laura Senior, USDA-Agricultural Research Services, for assistance with immunity assays.

\section{References}

[1] A. E. Hajek, "Ecology of terrestrial fungal entomopathogens," Advances in Microbial Ecology, vol. 15, no. 1, pp. 193-249, 1999.

[2] D. T. Gwynne, Katydids and Bushcrickets: Reproductive Behavior and Evolution of the Tettigoniidae, Cornell University Press, Ithaca, NY, USA, 2001.

[3] S. J. Simpson, G. A. Sword, P. D. Lorch, and I. D. Couzin, "Cannibal crickets on a forced march for protein and salt," Proceedings of the National Academy of Sciences of the United States of America, vol. 103, no. 11, pp. 4152-4156, 2006.

[4] B.-D. Sun, H.-Y. Yu, A. J. Chen, and X.-Z. Liu, "Insectassociated fungi in soils of field crops and orchards," Crop Protection, vol. 27, no. 11, pp. 1421-1426, 2008.

[5] A. E. Hajek and R. J. Leger, "Interactions between fungal pathogens and insect hosts," Annual Review of Entomology, vol. 39, pp. 293-322, 1994.

[6] L. M. Mullen and G. J. Goldsworthy, "Immune responses of locusts to challenge with the pathogenic fungus Metarhizium or high doses of laminarin," Journal of Insect Physiology, vol. 52, no. 4, pp. 389-398, 2006.

[7] S. N. Gardner and M. B. Thomas, "Costs and benefits of fighting infection in locusts," Evolutionary Ecology Research, vol. 4, no. 1, pp. 109-131, 2002.

[8] K. Wilson, M. B. Thomas, S. Blanford, M. Doggett, S. J. Simpson, and S. L. Moore, "Coping with crowds: densitydependent disease resistance in desert locusts," Proceedings of the National Academy of Sciences of the United States of America, vol. 99, no. 8, pp. 5471-5475, 2002.

[9] J. H. Turnbow, Temperature-sensitive Beauveria bassiana mycosis in the Mormon cricket, Anabrus simplex, M.S. thesis, Montana State University, Bozeman, MT, USA, 1998.

[10] J. P. Gillespie, C. Burnett, and A. K. Charnley, "The immune response of the desert locust Schistocerca gregaria during mycosis of the entomopathogenic fungus, Metarhizium anisopliae var acridum," Journal of Insect Physiology, vol. 46, no. 4, pp. 429-437, 2000.

[11] J. P. Gillespie and G. G. Khachatourians, "Characterization of the Melanoplus sanguinipes hemolymph after infection with Beauveria bassiana or wounding," Comparative Biochemistry and Physiology B, vol. 103, no. 2, pp. 455-463, 1992. 
[12] S. Y. Hung and D. G. Boucias, "Phenoloxidase activity in Hemolymph of Naive and Beauveria bassiana-infected Spodoptera exigua Larvae," Journal of Invertebrate Pathology, vol. 67, no. 1, pp. 35-40, 1996.

[13] D. G. Boucias, S. Y. Hung, I. Mazet, and J. Azbell, "Effect of the fungal pathogen, Beauveria bassiana, on the lysozyme activity in Spodoptera exigua larvae," Journal of Insect Physiology, vol. 40, no. 5, pp. 385-391, 1994.

[14] P. de Azambuja, E. S. Garcia, N. A. Ratcliffe, and J. David Warthen Jr., "Immune-depression in Rhodnius prolixus induced by the growth inhibitor, Azadirachtin," Journal of Insect Physiology, vol. 37, no. 10, pp. 771-777, 1991.

[15] E. Seyoum, D. Moore, and A. K. Charnley, "Reduction in flight activity and food consumption by the desert locust, Schistocerca gregaria, after infection with Metarhizium flavoviride," Zeitschrift für Angewandte Entomologie, vol. 118, no. 3, pp. 310-315, 1994.

[16] P. Dean, J. C. Gadsden, E. H. Richards, J. P. Edwards, A. K. Charnley, and S. E. Reynolds, "Modulation by eicosanoid biosynthesis inhibitors of immune responses by the insect Manduca sexta to the pathogenic fungus Metarhizium anisopliae," Journal of Invertebrate Pathology, vol. 79, no. 2, pp. 93101, 2002.

[17] R. B. Srygley, P. D. Lorch, S. J. Simpson, and G. A. Sword, "Immediate protein dietary effects on movement and the generalised immunocompetence of migrating Mormon crickets Anabrus simplex (Orthoptera: Tettigoniidae)," Ecological Entomology, vol. 34, no. 5, pp. 663-668, 2009. 

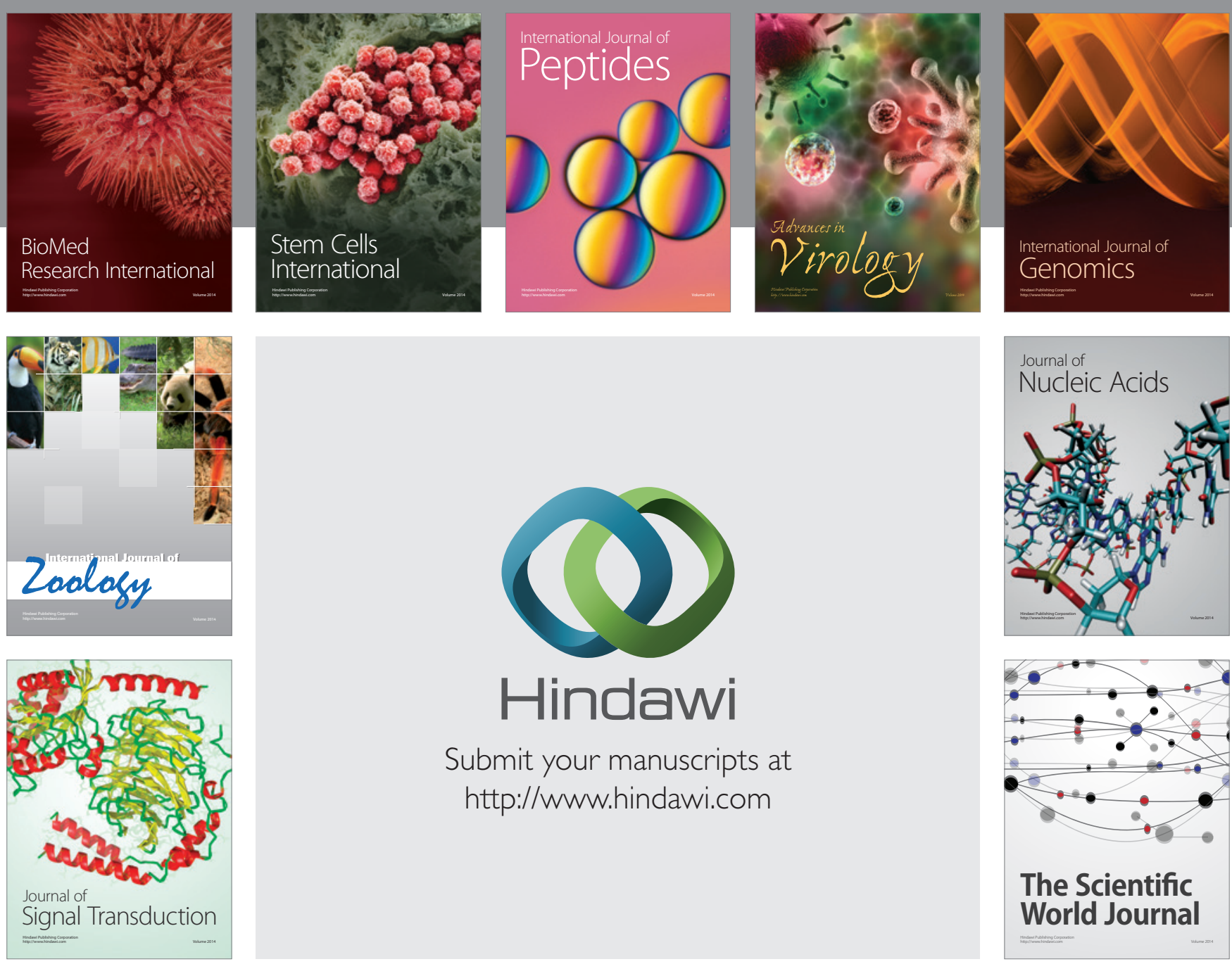

Submit your manuscripts at

http://www.hindawi.com
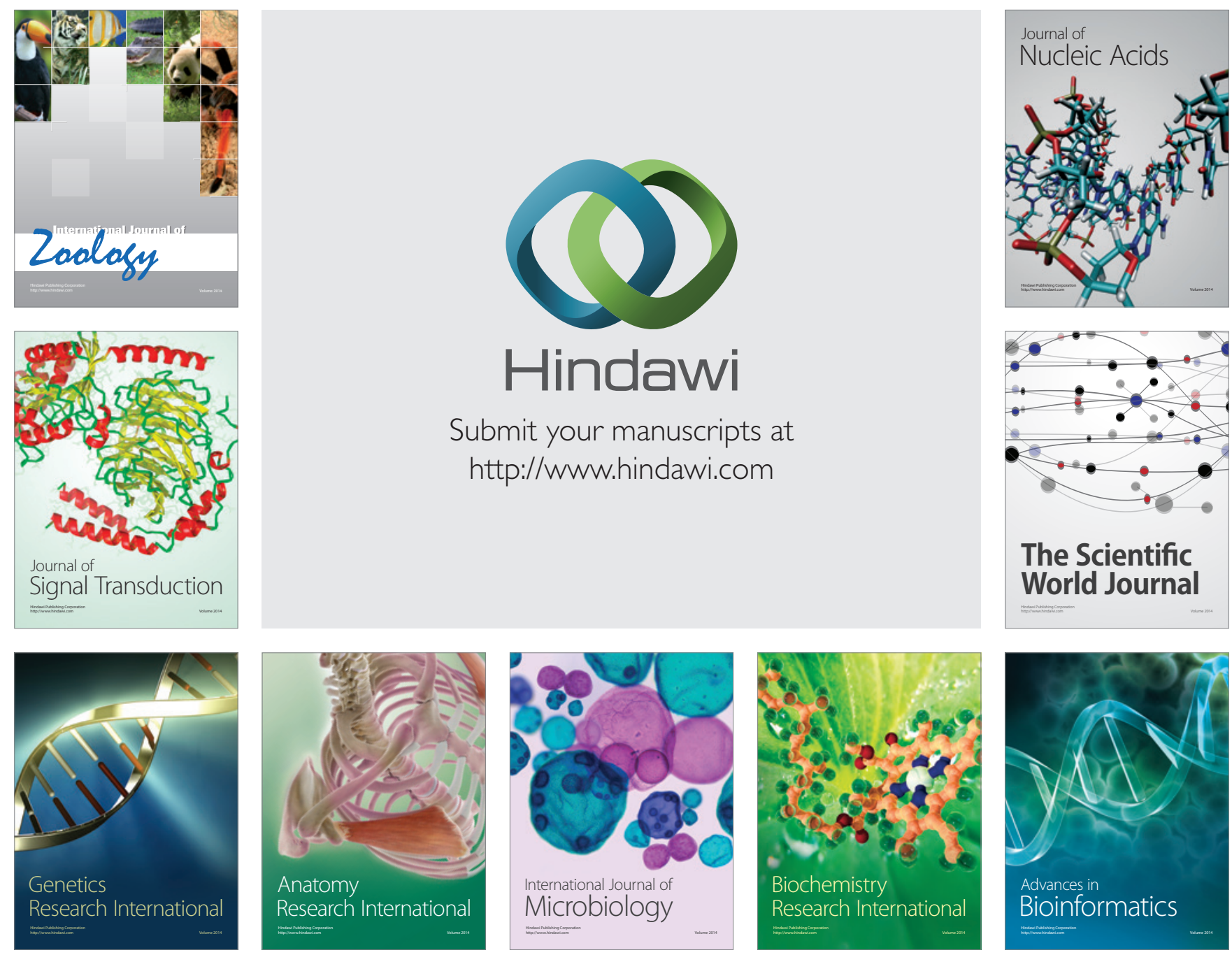

The Scientific World Journal
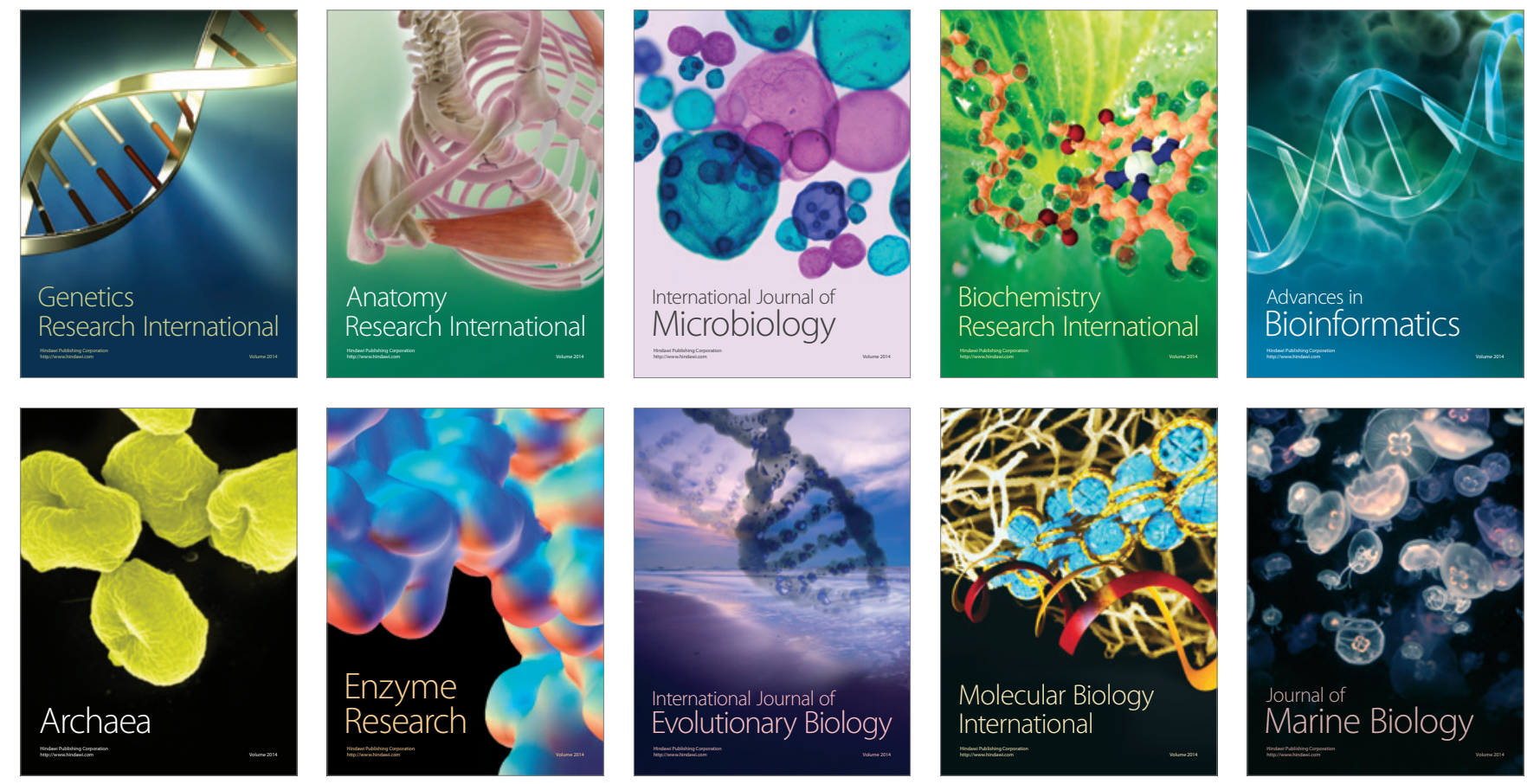